\title{
Thyroid disease
}

\section{Using diagnostic tools effectively}

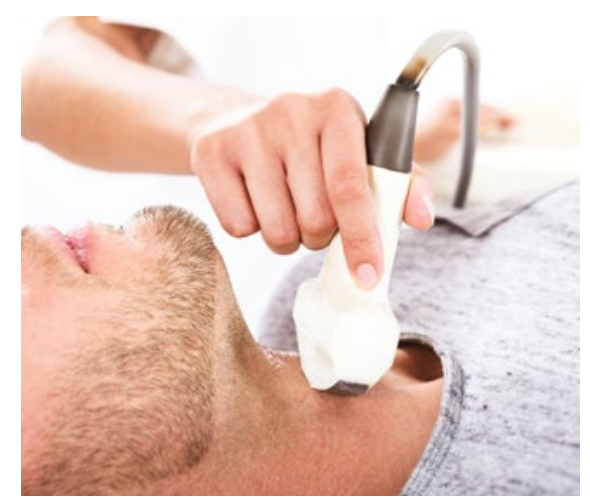

CPD

Emma E Croker, Shaun A McGrath, Christopher W Rowe

\section{Background}

Symptoms of thyroid disease are common, and patients often seek initial assessment from their general practitioners.

\section{Objective}

The aim of this article is to assist with identifying the appropriate sequence of investigations for thyroid disorders, and identify investigations with low diagnostic yield in certain clinical contexts.

\section{Discussion}

Common thyroid disorders - such as hypothyroidism, hyperthyroidism and thyroid nodules - require different sequences of investigations to assist with formulating a diagnosis and plan. Thyroid disorders are frequent in women of childbearing age and require a specialised approach. Awareness of less common thyroid conditions allows for individualised workup in these cases.
AUSTRALIAN DATA SHOW that thyroid tests are ordered at least once per every 100 problems managed in general practice. ${ }^{1}$ Of the five NPS MedicineWise 'Choosing Wisely' initiatives in endocrinology, two relate to thyroid investigation. ${ }^{2}$ It is important that suspected thyroid abnormalities are investigated appropriately to minimise patient anxiety, inefficiency and cost. This article provides succinct recommendations for the investigation of suspected thyroid disorders in adults.

\section{When should thyroid stimulating hormone be checked?}

Thyroid stimulating hormone (TSH)

should be checked:

- when screening for thyroid hormone excess or deficiency on the basis of symptoms or risk factors

- when goitre or thyroid nodules are identified

- when monitoring uncomplicated thyroxine replacement therapy, with a minimum interval of 4-6 weeks following a dose change to allow achievement of a steady state, and annually when stable

- prior to, and early in the first trimester of, pregnancy in women treated with levothyroxine or those with risk factors for thyroid dysfunction.
Measurement of TSH is a screening test for suspected thyroid dysfunction. TSH release from the anterior pituitary is tightly controlled by a negative feedback loop from the circulating thyroid hormones thyroxine (T4) and triiodothyronine (T3). A small rise in thyroid hormone production leads to a logarithmic suppression of TSH, and vice versa, meaning that TSH is a very sensitive marker of thyroid dysfunction. ${ }^{3}$ Assays for TSH are robust, and interference is uncommon. For these reasons, and because central thyroid dysfunction (pituitary or hypothalamic disorders) is rare, a normal TSH level largely excludes thyroid hormone excess or deficiency. ${ }^{4}$

Symptoms of hypothyroidism are non-specific and have poor correlation with thyroid hormone deficiency. Therefore, TSH is often used as a screening test in a wide range of clinical presentations with low pre-test probability for thyroid disease, including fatigue, metabolic syndrome and mental health disorders. With few exceptions, a TSH result within the normal range (generally 0.5-4.0 mIU/L, and reported for each assay) in a setting of low pre-test probability excludes thyroid dysfunction and generally does not require repeating for several years. ${ }^{5,6}$ If pre-test probability is high, a concordant measure of a normal FT4 with a normal TSH result confidently excludes incident thyroid dysfunction. In 
contrast, hyperthyroidism is commonly symptomatic, with higher clinical and biochemical correlation.

Once an abnormal TSH level is detected, it must be interpreted with reference to thyroid hormone levels. TSH is the driver hormone for intrathyroidal synthesis of the pro-hormone T4, which constitutes approximately $90 \%$ of the hormonal secretion from the thyroid, along with a small amount of T3. T4 is converted to the biologically active $\mathrm{T} 3$ by deiodinase enzymes. This physiology is important in the diagnosis and therapy of thyroid disorders (Figure 1).

In patients treated with levothyroxine, TSH is used to monitor adequacy of replacement. TSH testing should not be repeated until 4-6 weeks after a dose change to ensure a steady state is reached. ${ }^{7}$ Monitoring free T3 (FT3) is not helpful in this situation because it is cellular T3, not circulating $\mathrm{T} 3$, that is of biological relevance. Once the patient is stable on levothyroxine, TSH should be measured annually. Weight loss or gain, commencement or cessation of hormonal contraception/hormonal replacement, pregnancy or intercurrent illness can all change requirements for thyroid hormone and should prompt a review of TSH levels. ${ }^{8}$ The utility of universal testing of TSH in the first trimester of pregnancy has not been established and is best limited to patients with risk factors for thyroid dysfunction or those treated with levothyroxine. A TSH level of $>4 \mathrm{mIU} / \mathrm{L}$ is an agreed threshold for initiating levothyroxine in the first trimester. ${ }^{9}$ Initiating levothyroxine with TSH levels between $2.6 \mathrm{mIU} / \mathrm{L}$ and $4 \mathrm{mIU} / \mathrm{L}$ is controversial, as evidence for benefit is conflicting.

\section{How should an elevated TSH level be investigated?}

An elevated TSH level should be investigated in the following ways:

- If TSH is high, check TSH with free T4 (FT4)

- elevated TSH with FT4 below the reference range diagnoses primary hypothyroidism

- elevated TSH with FT4 within the reference range diagnoses mild or subclinical hypothyroidism.

- A mildly raised TSH will often resolve without treatment; therefore, thyroid function tests should generally be repeated at least once after 1-3 months before further investigation or treatment.

- Thyroid ultrasonography and thyroid scintigraphy should not be performed for uncomplicated hypothyroidism without a palpable nodule.

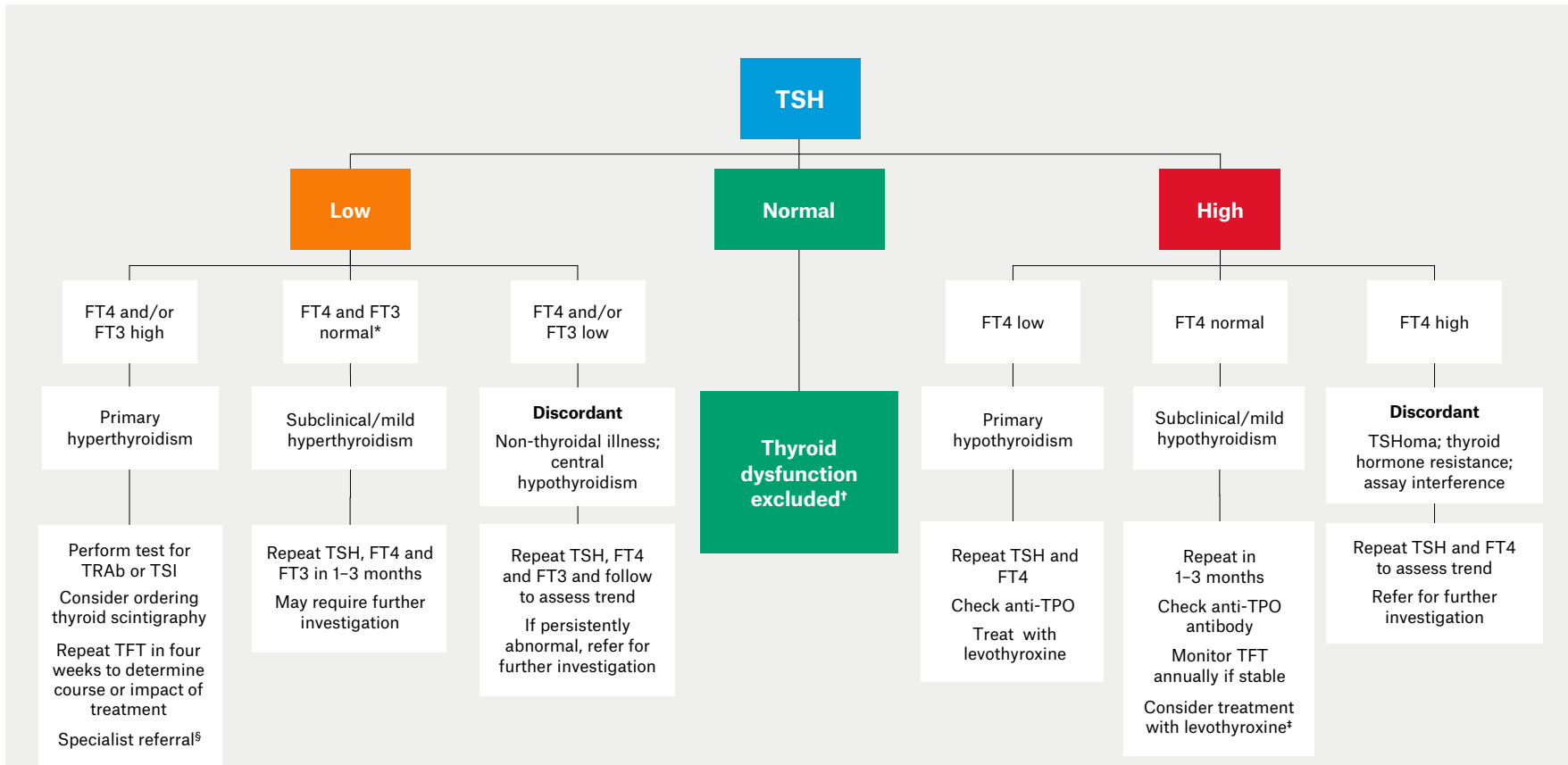

Figure 1. Investigation of abnormal TSH in non-pregnant adults

Note: Non-thyroidal illness can cause several patterns of thyroid hormone disturbance; however, the abnormalities are usually mild and resolve over time. Amiodarone can cause several patterns of thyroid dysfunction, and abnormal thyroid function tests within six months of amiodarone use require specialist evaluation.

*This pattern can be seen in non-thyroidal illness or rarely in central hypothyroidism

tIf suspicion of underlying pituitary or hypothalamic disease or specific factor affecting TSH interpretation, perform TSH, FT4 +/- FT3

${ }^{\ddagger}$ May require treatment, especially in younger patients and those planning pregnancy

s Specialist referral not required if transient thyroiditis

FT3, free triiodothyronine; FT4, free thyroxine; TFT, thyroid function test; TPO, thyroid peroxidase; TRAb, thyroid stimulating hormone receptor antibody;

TSH, thyroid stimulating hormone; TSHoma, thyrotropinoma; TSI, thyroid stimulating immunoglobulin 
Subclinical hypothyroidism implies absence of symptoms, but this biochemical abnormality can be associated with vague, non-specific symptoms and is perhaps better thought of as mild hypothyroidism. Making a diagnosis of subclinical or mild hypothyroidism, and commencing treatment, should not be rushed outside the setting of pregnancy or pregnancy planning. TSH and FT4 should be monitored for several months to determine a clear trend. Patient age, symptoms and comorbidities should all be weighed prior to considering levothyroxine therapy. ${ }^{10}$

In many situations, especially in mild cases with $\mathrm{TSH}<10 \mathrm{mIU} / \mathrm{L}$ or in the setting of recent illness, it is appropriate to repeat thyroid function tests in 4-6 weeks to exclude transient thyroid dysfunction. ${ }^{11}$

In pregnant women, $\mathrm{TSH}$ above the trimester-specific reference range (or $4 \mathrm{mIU} / \mathrm{L}$, if unavailable) should prompt immediate review and specialist referral. It is recommended that TSH and FT4 levels and thyroid peroxidase (TPO) antibodies are checked while commencing levothyroxine therapy, and thyroid function tests repeated fourth weekly until stable.

Antibodies to TPO and thyroglobulin are present in up to $18 \%$ of euthyroid individuals ${ }^{12}$ and do not require treatment in the presence of normal thyroid function. Once TPO or thyroglobulin antibodies are confirmed, levels do not need to be rechecked as this will not influence management. Patients with positive antibodies and mild or subclinical hypothyroidism progress to overt primary hypothyroidism at a rate of approximately $5 \%$ per year, with a much lower risk of progression in the setting of a normal TSH level. ${ }^{6}$

Ultrasonography or functional imaging of the thyroid is not indicated for investigation or diagnosis of routine primary hypothyroidism or positive TPO antibodies unless there is an obvious palpable solitary or dominant nodule.

\section{How should a suppressed TSH level be investigated?}

A suppressed TSH level should be investigated in the following ways:
- if TSH is low, check FT4 and FT3

- FT4 and/or FT3 above the reference range diagnoses primary hyperthyroidism

- a mildly low TSH (0.1-0.5 mIU/L) with normal free thyroid hormones suggests mild or subclinical hyperthyroidism, non-thyroidal illness or interference from other medications

- positive TSH receptor antibodies (TRAb; or thyroid stimulating immunoglobulins [TSI]) support a diagnosis of Graves' disease

- thyroid scintigraphy should be performed to distinguish between Graves' disease, toxic nodules and thyroiditis if the TRAb test is negative or there is diagnostic uncertainty

- thyroid ultrasonography is generally unhelpful in determining the cause of hyperthyroidism.

A low TSH level can be due to both thyroidal and non-thyroidal illness. Minor suppression of TSH (0.1-0.5 mIU/L) can be due to subclinical (or mild) hyperthyroidism, transient hyperthyroidism, non-thyroidal illness or medications that suppress TSH, and it will often resolve without treatment. In this situation, thyroid function tests should be monitored in the first instance, usually for between six weeks and three months, before further diagnostic workup is undertaken. ${ }^{13}$

Elevated TRAb levels are specific for Graves' disease and should be measured if the TSH is persistently suppressed below $0.1 \mathrm{mIU} / \mathrm{L}$, although can be negative in a small proportion of cases. ${ }^{14} \mathrm{TSI}$ testing is performed in some laboratories and is interpreted similarly. TPO and thyroglobulin antibodies are not usually helpful in distinguishing between aetiologies.

If the TRAb test is negative or if there is diagnostic uncertainty, Tc-99m pertechnetate thyroid scintigraphy is indicated for establishing the aetiology of hyperthyroidism in non-pregnant adults. ${ }^{13}$ The tracer uptake within thyrocytes corresponds with thyroid activity, as it mimics iodine uptake in the gland. Both the pattern of uptake, and a quantification of uptake (compared visually to salivary glands or reported as a percentage/ratio, as in Figure 2A) are useful. Tracer uptake is usually low (or absent) in thyroiditis, and for up to six weeks following iodinated contrast (Figure 2B). Autonomously functioning nodules appear as focal areas of increased uptake (Figure 2C). Graves' disease presents as diffuse and symmetrical uptake throughout both lobes (Figure 2D, where uptake can also be seen in the pyramidal lobe). Tc-99m pertechnetate is concentrated in breast milk; therefore, infants should not be breastfed for 26 hours after scintigraphy..$^{15}$ Thyroid scintigraphy is contraindicated in pregnancy.

Thyroid ultrasonography is not indicated for the routine evaluation of hyperthyroidism, as the findings generally do not distinguish between causes. This is because both thyroid nodules and autoimmune thyroid disease are common background pathologies, and the presence of features of those conditions does not confirm the aetiology of the biochemical disturbance.

\section{When else should FT4 and FT3 be checked?}

It is important to:

- check TSH, FT4 and FT3 to evaluate thyroid function in settings where TSH alone may be unreliable, such as - suspected pituitary or hypothalamic disease

- suspected assay interference

- rapidly changing thyroid function

- check FT4 (not TSH) to monitor and adjust levothyroxine replacement in patients with central hypothyroidism due to pituitary or hypothalamic disease

- note that measurement of reverse T3 is not recommended for the investigation of thyroid dysfunction.

TSH alone is insufficient to assess thyroid function in several situations: if hypothalamic or pituitary disease is suspected, or if assay interference is likely (eg because of high-dose biotin supplementation or heterophilic antibodies). It is important that clinicians are alert for discordant-appearing thyroid function tests (eg an inappropriately normal TSH result with low FT4 levels), which are the hallmark of pituitary dysfunction or assay interference. In 
the presence of significant symptoms/ signs of thyroid dysfunction, checking free thyroid hormones may assist with detecting rare cases of discordant thyroid function tests that require further investigation.

In patients treated with levothyroxine in the setting of pituitary or hypothalamic disease (secondary hypothyroidism), TSH levels must not be used to monitor adequacy of replacement, and reductions in levothyroxine on the basis of a low TSH level in this setting are incorrect. Instead, FT4 levels must be used to monitor and adjust levothyroxine.

In addition, monitoring TSH plus FT4/FT3 levels is required when initiating thionamide therapy (carbimazole or propylthiouracil) and in settings of rapidly changing thyroid function where changes in TSH can lag behind FT4 and FT3 (eg following radioactive iodine, thyroiditis or following a change in levothyroxine dose in early pregnancy).

Reverse $\mathrm{T} 3$ is an inactive metabolite of thyroxine and has no role in routine assessment of thyroid function.
When should thyroid ultrasonography be ordered?

Thyroid ultrasonography should be ordered:

- to investigate a structural thyroid abnormality (nodule or goitre), identified by palpation or as a coincidental finding on other imaging, noting that

- routine screening for thyroid nodules or thyroid cancer is not recommended

- TSH should be checked as the first investigation in patients with thyroid nodules

- to determine the risk of malignancy in known thyroid nodules

- prior to neck surgery for thyroid cancer, to evaluate for local tumour invasion and nodal metastases in the central and lateral neck

- following thyroid cancer surgery, to screen for local recurrence.

Thyroid ultrasonography is indicated to assess the extent of goitre and to stratify the risk of malignancy in thyroid nodules. Most thyroid nodules are benign and do not require biopsy or follow-up in the absence of clinical change. Ultrasound scoring systems assign a malignancy risk to nodules and determine whether follow-up ultrasonography or biopsy is required (Table 1 ). The American College of Radiology Thyroid Imaging Reporting and Data System (TI-RADS) ${ }^{16}$ and the American Thyroid Association sonographic pattern system are most commonly used in Australia. ${ }^{17}$

Fine-needle aspiration biopsy results are commonly reported using the Bethesda System for Reporting Thyroid Cytopathology. Biopsy results should be interpreted as part of a 'triple assessment' along with clinical context and sonographic features to determine appropriate management. Benign biopsy results (Bethesda II) should prompt follow-up surveillance ultrasonography according to sonographic risk (Table 1). Abnormal biopsy results, or biopsy results that are clearly discordant with sonographic risk, require specialist assessment.

Following the use of radioactive iodine for thyroid nodules, the common sonographic scoring systems are inaccurate and need to be interpreted with caution.
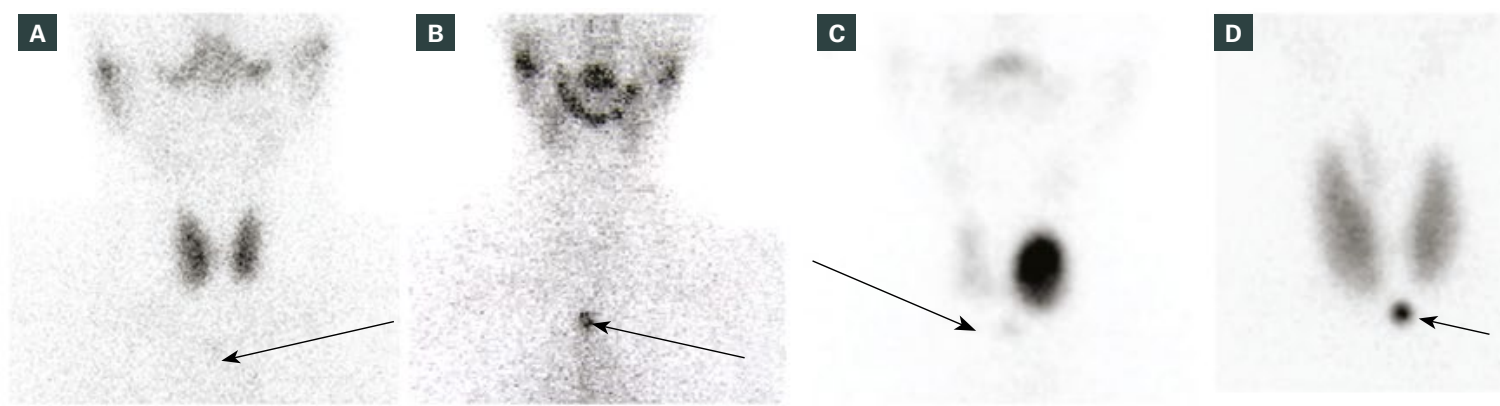

\begin{tabular}{lcccc} 
Diagnosis & Normal thyroid & Thyroiditis & Left toxic adenoma & Graves' disease \\
$\begin{array}{l}\text { Thyroid } \\
\text { uptake }\end{array}$ & Diffuse, symmetrical & Low or absent & $\begin{array}{c}\text { Increased in nodule } \\
\text { Contralateral reduced }\end{array}$ & Diffuse, symmetrical \\
$\begin{array}{l}\text { Salivary } \\
\text { gland uptake }\end{array}$ & Normal & Appears prominent & Appears reduced & Appears reduced \\
\hline
\end{tabular}

Figure 2. Anterior global views of Tc-99m pertechnetate thyroid scintigraphy. Salivary glands are visible in the upper neck of each figure but are seen only faintly in panels $C$ and $D$ as a result of the relative intensity of thyroidal uptake. Arrows indicate the position of the suprasternal notch with an overlying marker.

A. Normal scintigraphic appearance of thyroid; B. Thyroiditis, demonstrating absent uptake in expected position of thyroid gland, and relatively prominent uptake in salivary glands; C. Toxic adenoma in left lobe thyroid, with relative suppression of uptake in the contralateral thyroid; D. Graves disease. Uptake is diffuse and symmetrical in both lobes, and in the pyramidal lobe. Uptake is increased with reference to the salivary glands. 
Thus, routine ultrasonography following radioactive iodine is not recommended. Any clinically significant thyroid nodule following radioactive iodine is important and requires specialist review.
Screening asymptomatic individuals for thyroid cancer with thyroid ultrasonography is not recommended. It results in over-investigation of clinically insignificant thyroid nodules and an increased rate of surgery for thyroid cancer without any change in mortality. ${ }^{18,19}$ Screening for thyroid cancer could be considered in the rare cases of confirmed high-risk genetic

\section{Table 1. Sonographic risk stratification and follow-up of thyroid nodules according to American College of Radiology (ACR) Thyroid Imaging Reporting and Data System (TI-RADS) ${ }^{16,22}$}

\begin{tabular}{|c|c|c|c|c|}
\hline ACR TI-RADS & Definition & Risk of malignancy & Fine-needle aspiration biopsy & Follow-up ultrasonography \\
\hline TR2 & Not suspicious & $<2 \%$ & No & Not routine* \\
\hline TR3 & Mildly suspicious & $\sim 5 \%$ & $\begin{array}{l}\text { Yes, if } \geq 2.5 \mathrm{~cm} \\
\text { Otherwise perform follow-up } \\
\text { ultrasonography }\end{array}$ & One, three and five years ${ }^{\dagger}$ \\
\hline TR5 & Highly suspicious & $>20 \%$ & $\begin{array}{l}\text { Yes, if } \geq 1 \mathrm{~cm} \\
\text { Otherwise perform follow-up } \\
\text { ultrasonography }\end{array}$ & Yearly for five years $^{\dagger}$ \\
\hline
\end{tabular}

*Repeat ultrasonography at 1-2 years could be considered to confirm initial assessment.

tIf there is no clinically significant growth (20\% increase in two nodule dimensions and a minimum increase of 2 mm) and no change in TR category after five years, follow-up can be discontinued. Repeating thyroid ultrasonography more frequently than yearly is not recommended.

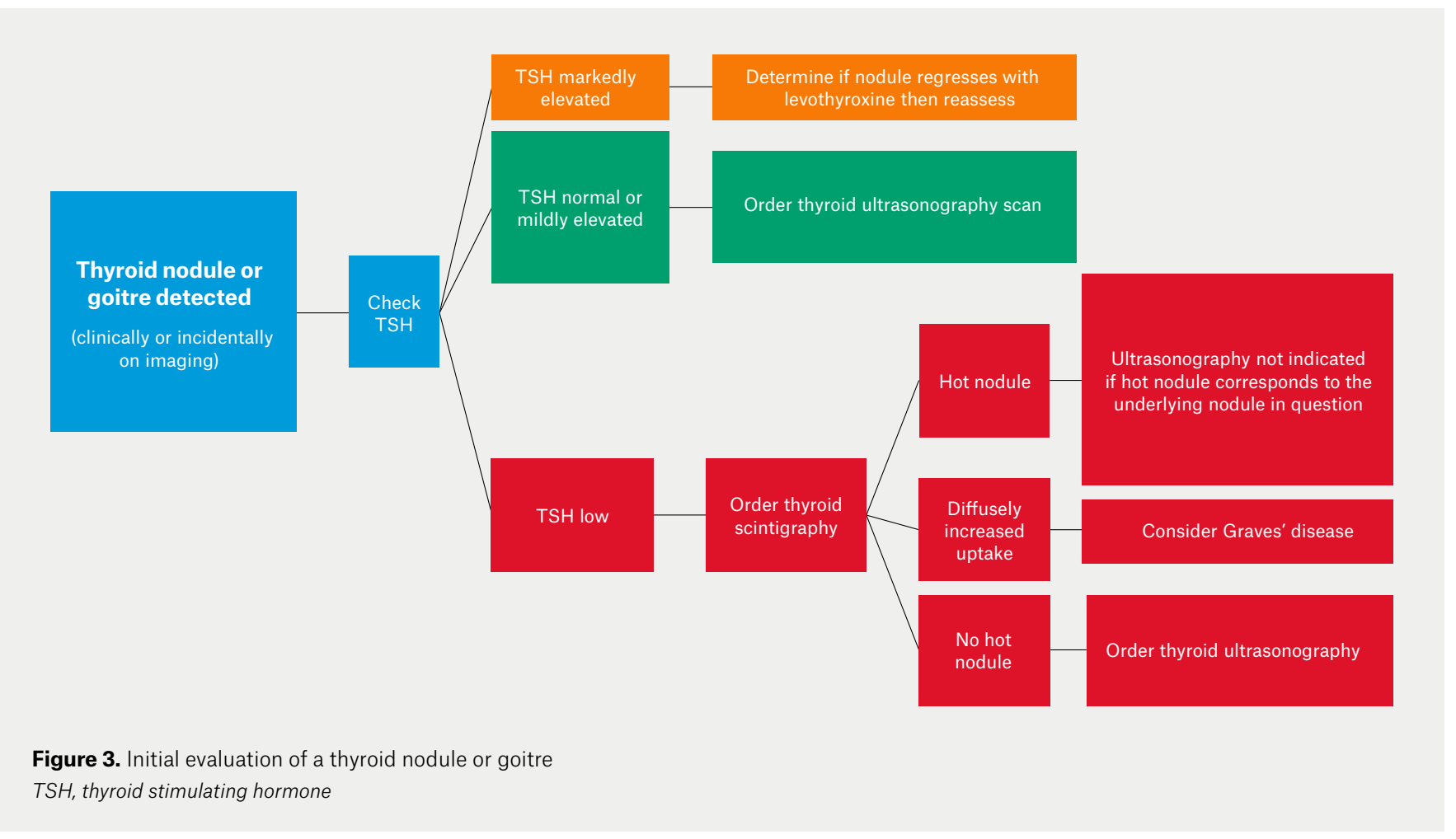


mutations or early childhood exposure to ionising radiation. ${ }^{20,21}$

Functional thyroid nodules (eg toxic adenoma) have a very low risk of malignancy and do not require sonographic assessment for further risk stratification. Therefore, a low TSH level should prompt scintigraphy as the first investigation of thyroid nodules (Figure 3). A palpable nodule that is not clearly hyper-functioning on scintigraphy requires sonographic assessment, as it may be caused by a solid, discrete nodule (higher risk of malignancy) or simple cyst (benign).

Thyroid ultrasonography is not indicated, or listed on the Medicare Benefits Schedule, for the diagnostic workup of uncomplicated hypothyroidism, thyroid autoantibodies or neck pain in the absence of a known thyroid nodule.

\section{Conclusion}

Rational investigation of thyroid disease requires careful avoidance of over-investigation of minor abnormalities balanced with prompt diagnosis of serious health conditions. An awareness of which thyroid tests are appropriate or not indicated in different contexts will improve patient care and health resource utilisation.

\section{Key points}

- A normal TSH level excludes thyroid dysfunction in most situations.

- FT4 levels must be checked in the rare cases of underlying or suspected pituitary disease, and to guide levothyroxine replacement in patients with pituitary/hypothalamic disease.

- Anti-TPO and anti-thyroglobulin antibody levels do not need to be serially monitored.

- Thyroid ultrasonography should be ordered to investigate structural abnormalities in the thyroid.

- Thyroid ultrasonography is not indicated in the diagnostic workup of uncomplicated hypothyroidism or positive TPO/thyroglobulin antibodies.

- Thyroid scintigraphy should be performed to investigate the underlying aetiology of hyperthyroidism.

\section{Authors}

Emma E Croker BMed (Dist), Endocrine Advanced Trainee, Department of Endocrinology and Diabetes, John Hunter Hospital, NSW

Shaun A McGrath BSc (Med), MBBS (Hons I), FRACP, Visiting Medical Officer Endocrinologist, Department of Endocrinology and Diabetes, John Hunter Hospital, NSW; Conjoint Lecturer, School of Medicine and Public Health, University of Newcastle, NSW; Endocrinologist, Newcastle Endocrinology, NSW Christopher W Rowe BSc (Med), MBBS (Hons I), PhD, FRACP, Staff Specialist Endocrinologist, Department of Endocrinology and Diabetes, John Hunter Hospital, NSW; Conjoint Senior Lecturer, School of Medicine and Public Health, University of Newcastle, NSW; Endocrinologist, Newcastle Endocrinology, NSW Competing interests: None.

Funding: None.

Provenance and peer review: Commissioned, externally peer reviewed.

\section{Correspondence to:}

christopher.rowe@health.nsw.gov.au

\section{References}

1. Bayram C, Valenti L, Britt $H$. Orders for thyroid function tests: Changes over 10 years. Aust Fam Physician 2012;41(8):555.

2. Choosing Wisely Australia. The endocrine society of Australia. Surry Hills, NSW: NPS MedicineWise, 2017. Available at www.choosingwisely.org.au/ recommendations/esa [Accessed 18 November 2020].

3. van Deventer HE, Mendu DR, Remaley AT, Soldin SJ. Inverse log-linear relationship between thyroid-stimulating hormone and free thyroxine measured by direct analog immunoassay and tandem mass spectrometry. Clin Chem 2011;57(1):122-27. doi: 10.1373/ clinchem.2010.154088.

4. Schneider C, Feller M, Bauer DC, et al. Initial evaluation of thyroid dysfunction - Are simultaneous TSH and fT4 tests necessary? PloS One 2018;13(4):e0196631. doi: 10.1371/journal. pone.0196631.

5. Danese MD, Powe NR, Sawin CT, Ladenson PW. Screening for mild thyroid failure at the periodic health examination: A decision and costeffectiveness analysis. JAMA 1996;276(4):285-92 doi: 10.1001/jama.1996.03540040029029.

6. Huber G, Staub JJ, Meier C, et al. Prospective study of the spontaneous course of subclinical hypothyroidism: Prognostic value of thyrotropin, thyroid reserve, and thyroid antibodies. J Clin Endocrinol Metab 2002;87(7):3221-26. doi: 10.1210/jcem.87.7.8678.

7. Colucci P, Yue CS, Ducharme M, Benvenga S. A review of the pharmacokinetics of levothyroxine for the treatment of hypothyroidism. Eur Endocrinol 2013;9(1):40-47. doi: 10.17925/ EE.2013.09.01.40.

8. Duntas LH, Jonklaas J. Levothyroxine dose adjustment to optimise therapy throughout a patient's lifetime. Adv Ther 2019;36(Suppl 2):30-46. doi: 10.1007/s12325019-01078-2.

9. Hamblin PS, Sheehan PM, Allan C, et al. Subclinical hypothyroidism during pregnancy: The Melbourne public hospitals consensus. Intern Med J 2019;49(8):994-1000. doi: 10.1111/imj.14210.

10. Peeters RP. Subclinical hypothyroidism. N Eng J Med 2017;376(26):2556-65. doi: 10.1056/ NEJMcp1611144.

11. Chaker L, Bianco AC, Jonklaas J, Peeters RP. Hypothyroidism. Lancet 2017;390(10101):1550-62. doi: 10.1016/S0140-6736(17)30703-1.
12. Hollowell JG, Staehling NW, Flanders WD, et al. Serum TSH, T(4), and thyroid antibodies in the United States population (1988 to 1994): National health and nutrition examination survey (NHANES III). J Clin Endocrinol Metab 2002;87(2):489-99. doi: 10.1210/jcem.87.2.8182.

13. Ross DS, Burch HB, Cooper DS, et al. 2016 American thyroid association guidelines for diagnosis and management of hyperthyroidism and other causes of thyrotoxicosis. Thyroid 2016;26(10):1343-421. doi: 10.1089/thy.2016.0229.

14. Tozzoli R, Bagnasco M, Giavarina D, Bizzaro N TSH receptor autoantibody immunoassay in patients with Graves' disease: Improvement of diagnostic accuracy over different generations of methods. Systematic review and meta-analysis. Autoimmun Rev 2012;12(2):107-13. doi: 10.1016/j. autrev.2012.07.003.

15. Australian Radiation Protection and Nuclear Safety Agency. Radiation protection in nuclear medicine. Yallambie, Vic: ARPANSA, 2008.

16. Tessler FN, Middleton WD, Grant EG, et al. ACR thyroid imaging, reporting and data system (TI-RADS): White paper of the ACR TI-RADS committee. J Am Coll Radiol 2017;14(5):587-95. doi: 10.1016/j.jacr.2017.01.046.

17. Singh Ospina N, Iñiguez-Ariza NM, Castro MR Thyroid nodules: Diagnostic evaluation based on thyroid cancer risk assessment. BMJ 2020;368:I6670. doi: 10.1136/bmj.16670.

18. Ahn HS, Kim HJ, Welch HG. Korea's thyroidcancer 'epidemic' - Screening and overdiagnosis. N Engl J Med 2014;371(19):1765-67. doi: 10.1056/ NEJMp1409841.

19. Brito JP, Morris JC, Montori VM. Thyroid cancer: Zealous imaging has increased detection and treatment of low risk tumours. BMJ 2013;347:f4706. doi: 10.1136/bmj.f4706.

20. Haugen BR, Alexander EK, Bible KC, et al. 2015 American Thyroid Association management guidelines for adult patients with thyroid nodules and differentiated thyroid cancer: The American Thyroid Association guidelines task force on thyroid nodules and differentiated thyroid cancer. Thyroid 2016;26(1):1-133. doi: 10.1089/ thy.2015.0020.

21. Grossman DC, Curry SJ, Barry MJ, et al. Screening for thyroid cancer: US preventive services task force recommendation statement. JAMA 2017;317(18):1882-87. doi: 10.1001/jama.2017.4011.

22. Shen Y, Liu M, He J, et al. Comparison of different risk-stratification systems for the diagnosis of benign and malignant thyroid nodules. Front Oncol 2019:9:378. doi: 10.3389/fonc.2019.00378. 